\title{
EXPERIMENTAL DYNAMIC CHARACTERIZATION OF A RECONFIGURABLE ADAPTIVE PRECISION TRUSS
}

\author{
J. D. Hinkle and L. D. Peterson ${ }^{\dagger}$ \\ Center for Aerospace Structures \\ and Department of Aerospace Engineering Sciences \\ University of Colorado at Boulder \\ Boulder, CO 80309-0429
}

\begin{abstract}
The dynamic behavior of a reconfigurable adaptive truss structure with nonlinear joints is investigated. The objective is to experimentally examine the effects of the local nonlinearities on the global dynamics of the structure. Amplitude changes in the frequency response functions are measured at micron levels of motion. The amplitude and frequency variations of a number of modes indicate a nonlinear Coulomb friction response. Hysteretic bifurcation behavior is also measured at an amplitude approximately equal to the specified freeplay in the joint. Under the $1 \mathrm{~g}$ preload, however, the nonlinearity was dominantly characteristic of Coulomb friction with little evidence of freeplay stiffening.
\end{abstract}

\section{INTRODUCTION}

Future small spacecraft structures must be designed to be deployed while retaining the structural rigidity necessary for precision instrumentation. In contrast with most erectable spacecraft structures which have been investigated over the past fifteen years, small, compact structures are complex, nonlinear, time-variable dynamical systems. To successfully control the dynamics of these structures, it will be necessary to obtain high fidelity models based on ground test experiments. Unfortunately, there is little experience with system identification methods which are successful for nonlinear, time-varying structures. Furthermore, little is known about the confidence with which these structures can be modeled using existing finite element codes.

Examples of precision deployable structure concepts were assembled by Mikulas and Withnell [1]. Effective design of this type of structure will require a more thorough experimental background in deployable structural dynamics with a particular focus on nonlinear joint behavior.

\footnotetext{
* Graduate Research Assistant, Student Member AIAA

${ }^{\dagger}$ Assistant Professor. Senior Member AIAA, Associate Member ASME
}

Previous research in nonlinear joint effects on space structure dynamics included examinations of modal coupling effects (Sarver and Crawley [2]), and attempts to develop equivalent beam finite element models using an equivalent energy approach (Webster and Velde [3]). Bowden [4] examined the effects of a number of simple nonlinear joint models of on global beam and truss dynamics.

Tests on deployable, erectable, and rotary modules in the Middeck 0-gravity Dynamics Experiment (MODE) provided data on nonlinear variations in frequency, amplitude, and damping as functions of input amplitude, suspension stiffness, and preload [5]. A primaryconclusion from this testing was a general rule of one percent variance in the experimental determination of frequency and one-half percent error in that of damping for testing of this type of structure.

A significant difference between the MODE test structures and the batten actuated truss (BAT) being studied here is the shapes of the lowest modes of the structures. The beam-like behavior of the MODE test structures fails to exemplify the unique dynamic displacements which result from the variety of geometries found in more compact deployable structures such as precision reflectors and BAT. Unfortunately, the strong dependence of the dynamics of these structures on local displacements serves to increase the influence of local sources of nonlinearity on the global dynamics.

Initial results from force-state mapping experimentation on the pin-clevis joints used in BAT have revealed extremely complex behavior[6]. As a result, accurate modeling of these elements may require multiple degrees of freedom in addition to nonlinear considerations.

The experiment was organized as follows. Initially, multiple modal surveys of the structure are performed across the structure's deployment range. Then, to obtain piecewise constant models of the structure, the data was modeled by the Eigensystem Realization Algorithm (ERA) [7] as well as the Common Basis-normalized Structural Identification (CBSI) procedure [8]. These results were then compared with the predictions of a NASTRAN finite element model. In addition, a study of phase plane responses of the structure as a function of input amplitude and frequency is performed. 


\section{THE BATTEN-ACTUATED TRUSS}

Our experiments were performed on the batten actuated deployable truss depicted in Figures 1-4. Six screw jack actuators driven by $0.52 \mathrm{lb}$ motors control the motion from the packaged to fully deployed state. By independently changing the length of the screw jack actuators, the truss can be reconfigured with three degrees of freedom. The bearings and all mechanism components of this structure are preloaded by opposing springs to eliminate backlash and freeplay in the structure. As a result, it is a precision, reconfigurable adaptive truss capable of erectable structure precision. Throughout these tests, the truss was cantilevered from a backstop with two linear bearing connections allowing deployment.

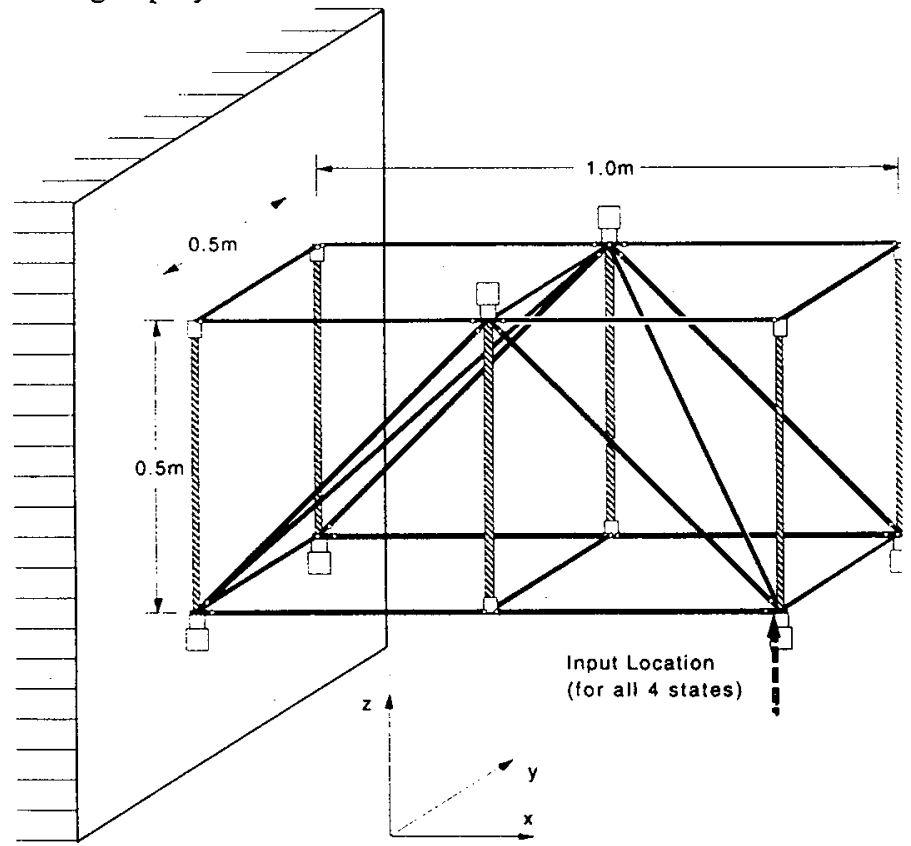

Figure 1: Reconfigurable Truss in Deployed State

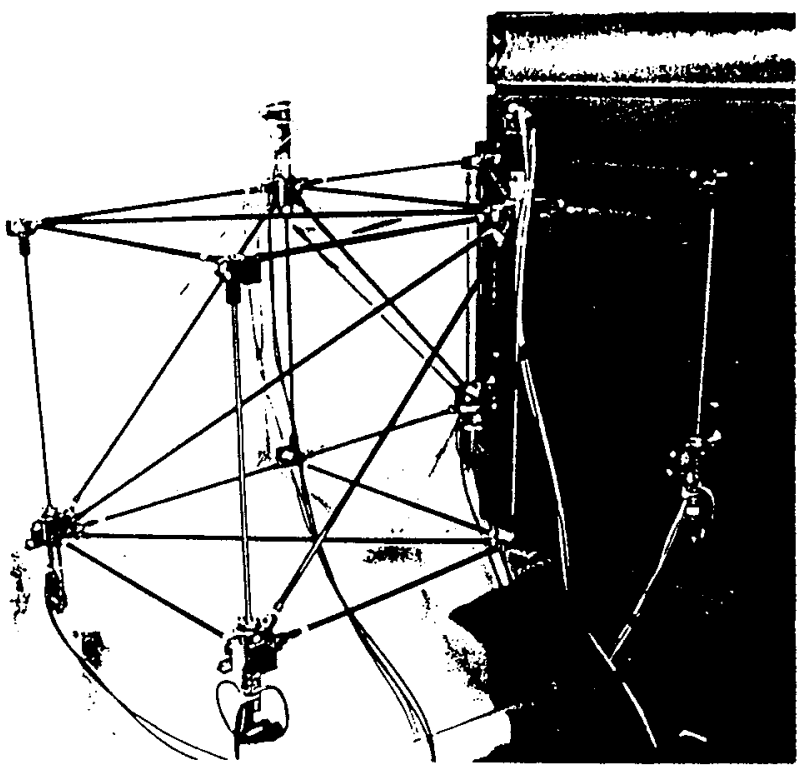

Figure 2: Photograph of Reconfigurable Truss in Deployed State
Stainless steel and aluminum pin-clevis hinges with bronze fittings connect the members. These nodes are the primary source of nonlinearity in the structure. The truss members are connected to the hinges with a shoulder bolt mounted to the hinge and a compression nut that clamps the member and shoulder bolt together. It should be noted that these hinge-member connections are identical to those used in other highly linear erectable truss hardware and most likely did not contribute to the observed nonlinear behavior.

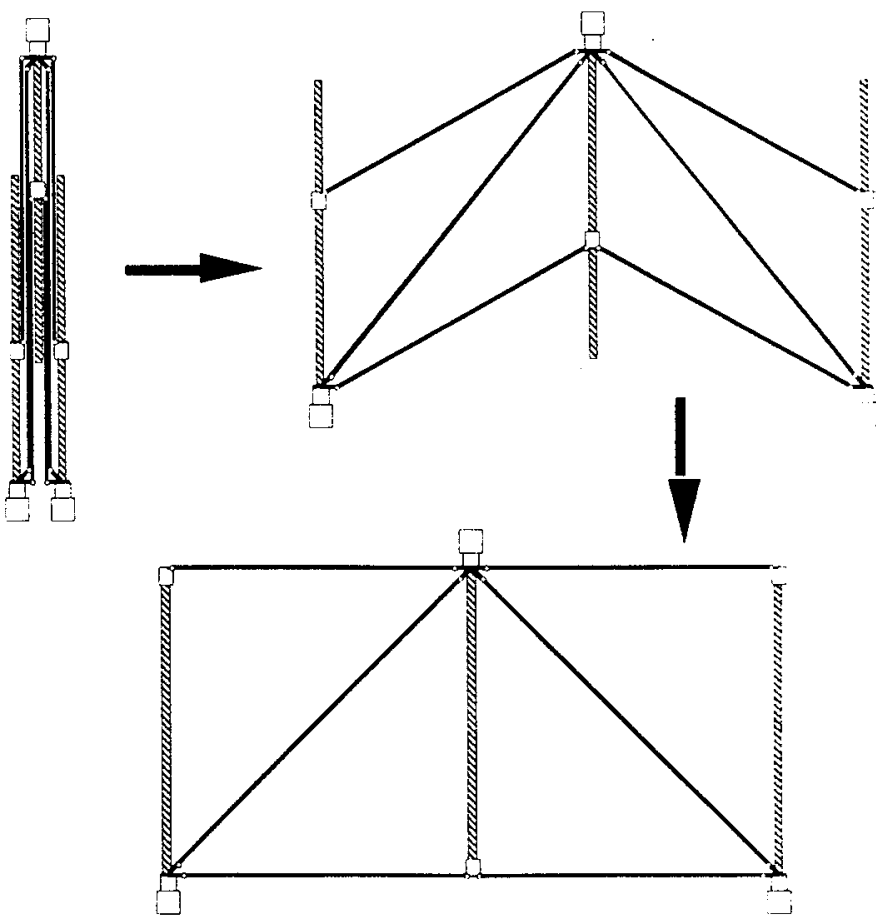

Figure 3: Batten Actuated Deployment

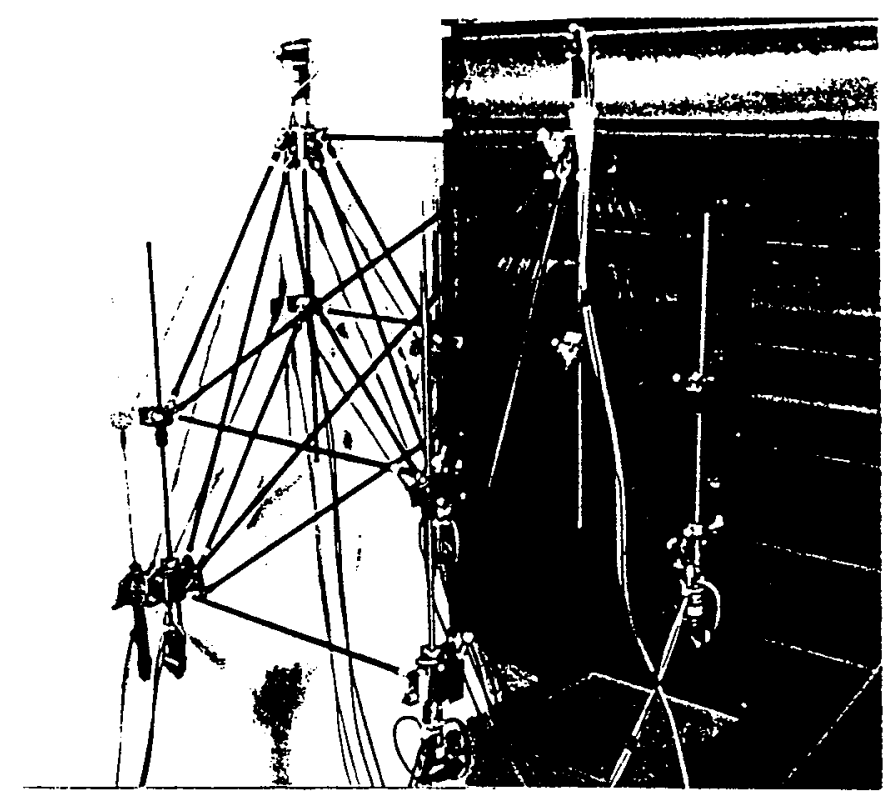

Figure 4: Photograph of Reconfigurable Truss in Half Deployed State 


\section{MODELING}

Finite element models of the structure were created in MSC/ NASTRAN [9] for each of the four tested deployment states. The models consisted solely of linear CBAR, RBAR, and point mass elements assembled to accurately represent the geometry of the structure. Each member was meshed into five CBAR elements and the inertial contributions of the motors were modeled as point masses offset from the appropriate nodes. The pin-clevis joints were modeled as y rotational degrees of freedom connections to the appropriate members. These connections were offset from the node centers by rigid members.

\section{SYSTEM IDENTIFICATION PROCEDURE}

\section{Experimental Procedure}

An initial bandwidth of interest of $0-200 \mathrm{~Hz}$ was selected and testing was performed at a $500 \mathrm{~Hz}$ sample rate to provide a safe Nyquist value. The high bandwidth is necessary to obtain high fidelity identified models for use in control, but increases the effect of dynamic nonlinearity on the identification. A pseudo burst-random input signal was used across $80 \%$ of a sixteen second window. Expecting nonlinear response characteristics, the test case matrix included four approximately logarithmically separated input amplitudes.

Sets of 300 trials were performed for each input amplitude at four equally spaced stages of deployment. The spectral data from these trials was averaged for each case to reduce the standard deviation of the data.

Along with collocated force and acceleration measurements at the input location, tri-axial acceleration measurements were made at each of the nodes. Additional pairs of vertically oriented accelerometers were located on the motorized joints to capture the "rocking" behavior of the joints depicted later. These led to a total of 54 acceleration output channels.

\section{Data Analysis Procedure}

Frequency response functions were generated for individual input-output pairs for each input amplitude. This data was then compared between deployment state cases.

The pseudo burst random FRFs were then curve fit using the time domain ERA system identification technique. The ERA Hankel matrix had 40 block rows and 1000 block columns. The choice of the number of block rows meant that the first $0.08 \mathrm{sec}-$ onds of each impulse response was used, ensuring that at least two full periods of the lowest mode of interest was used in the identification. The number of block columns was as large as feasible given computation time constraints.

These specifications were applied to identification procedures for models of order 300 to 20 to examine the sensitivity of the modal parameters of interest to the truncation order of the model. The results proved to be fairly consistent for models with 80 to 20 degrees of freedom. A statistical analysis of this data was then performed to quantify the amount of error expected from the model and assure that it was sufficiently less then the trends that were being observed. Trends in the identified modal frequencies and damping ratios as input amplitude increased are then examined for a pair of consistently identified modes.

\section{PHASE PLANE RESPONSE INVESTIGATION PRO- CEDURE}

Although pseudo burst-random is a common modal excitation waveform for a full bandwidth survey, it can mask the effects of nonlinearity. Consequently, we also investigated sine dwell resonance tests to examine the nonlinear boundaries of a number of modes.

This portion of the investigation was performed in a manner similar to the modal survey with the input provided by the shaker and outputs by the collocated input and output force and acceleration transducers used in the initial testing. A constant sinusoidal input was provided by a waveform generator. The phase plane response of the collocated input-output transfer function was then examined on an oscilloscope. In this manner, dependence of the phase plane response of the structure was measured as a function of input amplitude at a variety of modal resonance frequencies.

\section{RESULTS}

\section{Linear Vibration}

Some modal predictions from the finite element model are presented in Table 1. The initially surprising result was that the model predicts a softer response from the fully deployed state of the structure. This is more easily understood once one considers the shapes of the modes developed by the model (see Figures 5 $\& 6$ ). From these shapes, we notice that the greatest deflections of the structure occur with rotations of the mid-plane nodes allowing a pin-pin deflection shape of the screw-jack members. There is little torsional stiffness in the y direction about these nodes due to the degrees of freedom of all of the pin-clevis joints removing the y direction torsional stiffness contributions of the connected members. This lack of stiffness leads to the shapes of the first few lowest modes with larger deflections developing in the rightmost batten plane for the following modes. 
Table 1: Modeled and Measured Modal Frequencies

\begin{tabular}{|l|l|l|l|l|l|l|}
\hline $\begin{array}{c}\text { Mode } \\
\#\end{array}$ & \multicolumn{5}{|c|}{ Frequency (Hz) } \\
\hline & \multicolumn{3}{|c|}{ Half Deployment } & \multicolumn{3}{c|}{ Full Deployment } \\
\hline & Modeled & Measured & Error & Modeled & Measured & Error \\
\hline 1 & 39.0 & 31.4 & +7.6 & 37.8 & 42.7 & -4.9 \\
\hline 2 & 45.4 & 42.1 & +3.3 & 39.7 & 44.8 & -5.1 \\
\hline 3 & 52.5 & 46.7 & +5.8 & 50.2 & 53.0 & -2.8 \\
\hline
\end{tabular}

The result of this is that the shortening of the screw-jack members at half deployment serves to stiffen the softest point in the structure. Therefore, even with the weaker geometry of the half-deployed configuration, the overall stiffness of the structure is increased. These extreme node rotations are examples of the atypical low frequency displacements to be found in compact deployable structures.

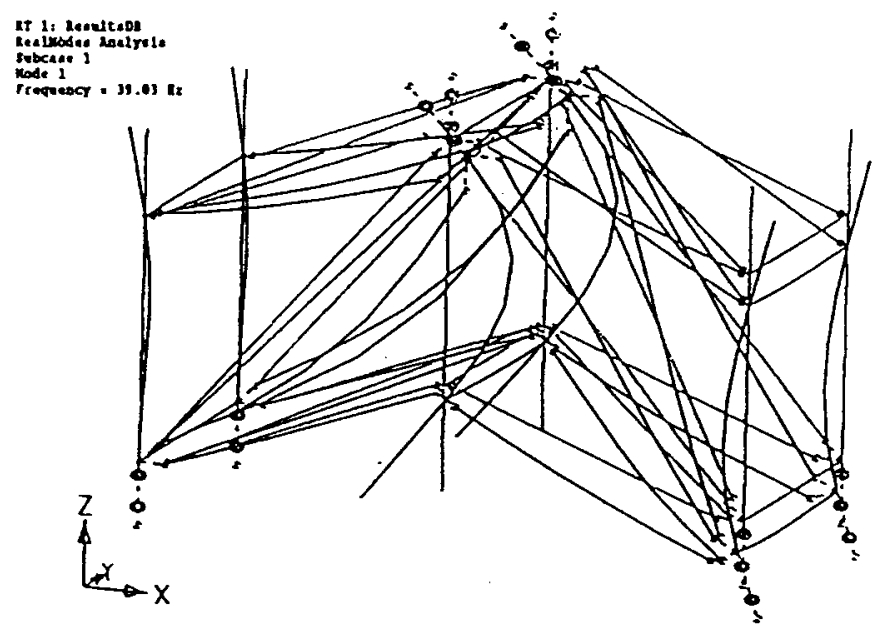

Figure 5: First Mode of Half Deployed Structure

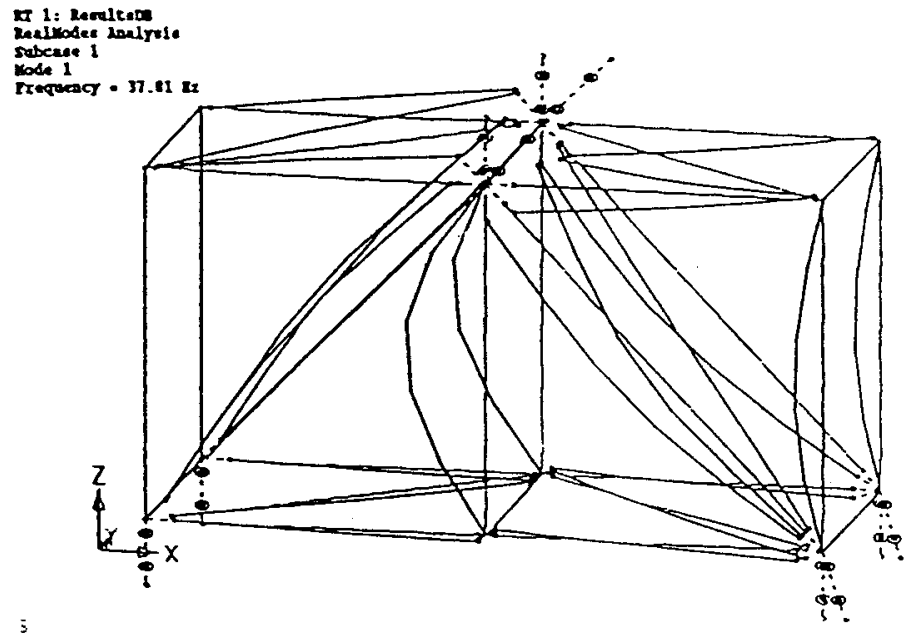

Figure 6: First Mode of Fully Deployed Structure

One of the first areas to be analyzed in the frequency response of the structure was the accuracy of the predicted stiffening of the structure at half deployment. In Table 1, we see that this is not supported by the modal test results. Also, in Figure 7, we observe a general softening in the measured response of the structure from full to half deployment. Evidence for this softening includes decreases in the frequencies of the lowest modes as well as increases in their amplitudes. Possible explanations for the inaccuracy of the models include the complexity of the geometry of the structure as well as failure to include the nonlinear behavior of the joints in the models.
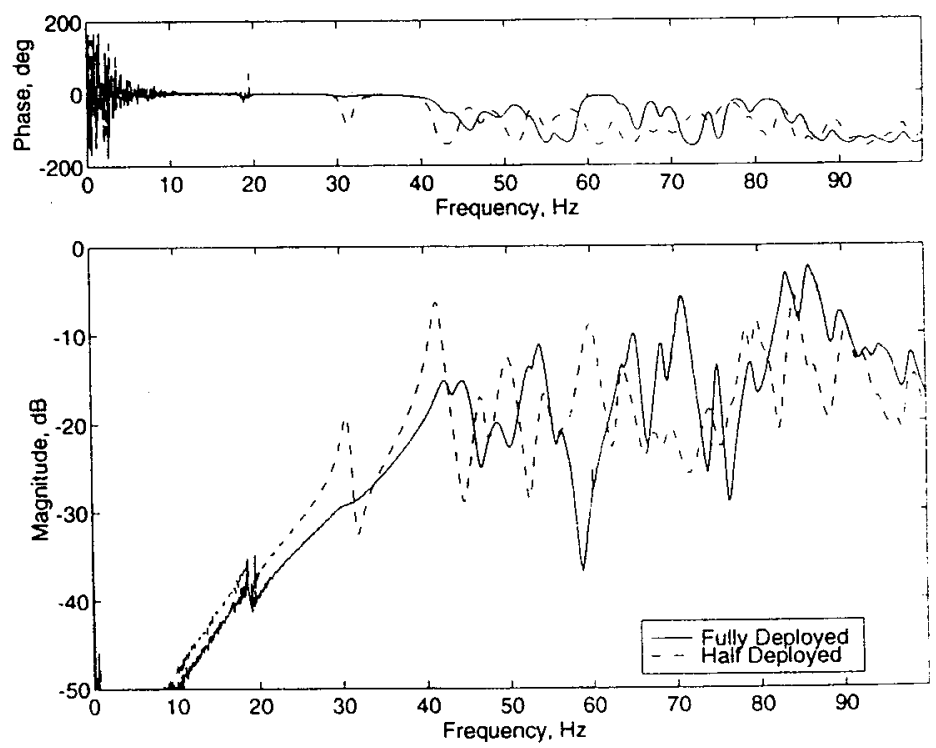

Figure 7: FRFs of Fully Deployed and Half Deployed Structure 


\section{Nonlinear Results}

Figure 8 displays the frequency responses of the fully deployed structure for all four input amplitudes. Comparison of these responses provides immediate evidence of the high level of nonlinearity in the structure. Trends to be observed include varying amounts of softening (reduced frequency), increased damping, and decreasing response amplitudes as input amplitude is increased. As seen in Bowden's study of fundamental nonlinear joint models [4], decreasing modal frequencies following increasing input amplitude are characteristic of a Coulomb friction nonlinearity. This is in contrast to the nature of joint freeplay dynamics acting to stiffen the dynamic response as input amplitude is increased.

In order to quantify the frequency and damping variations across all of the inputs, it was necessary to focus on modes that were best identified. This was a result of the highly nonlinear response for the highest input amplitude. Even allowing this, the identification algorithm was able to regularly identify only one of the selected modes for the highest input amplitude case. The mean identified modal parameter values for model sizes from 20 to 80 degrees of freedom is presented in Table 2 and in Figures 9 and 10.

The identified modal frequencies decreased by up to $2.7 \mathrm{~Hz}$ from the lowest to highest input amplitudes for the two selected modes. This variation was a nearly linear function of the input amplitude. The identified frequencies for mode $\mathrm{A}$ decreased at a rate of about $0.22 \mathrm{~Hz} / \mathrm{N}$, while mode $\mathrm{B}$ decreased at about 0.53 $\mathrm{Hz} / \mathrm{N}$.

As seen in Figure 10, identified damping ratios for the two modes revealed a slightly less consistent linear dependence on input amplitude. While the identified damping ratio for mode $A$ decreased slightly initially, both modes eventually experienced approximately a $140 \%$ increase in damping from the lowest to highest input amplitudes.

In an attempt to better understand the dynamic displacements of the structure, the displacements involved in these modes were simulated by the model identification software. The motion of the measured points resembled global bending modes for modes $\mathrm{A}$ and $\mathrm{B}$.

Table 2: Trends in Modal Parameters

\begin{tabular}{|l|l|l|l|l|}
\hline \multirow{2}{*}{ Input Amplitude(N) } & \multicolumn{2}{|c|}{ Mode A } & \multicolumn{2}{c|}{ Mode B } \\
\cline { 2 - 5 } & $\omega(\mathrm{Hz})$ & $\zeta(\%)$ & $\omega(\mathrm{Hz})$ & $\zeta(\%)$ \\
\hline 0.89 & 86.0 & 1.17 & 83.7 & 1.15 \\
\hline 2.23 & 85.7 & 0.97 & 82.7 & 1.75 \\
\hline 4.45 & 84.8 & 1.11 & 81.8 & 2.90 \\
\hline 13.3 & 83.28 & 2.83 & NA & NA \\
\hline
\end{tabular}
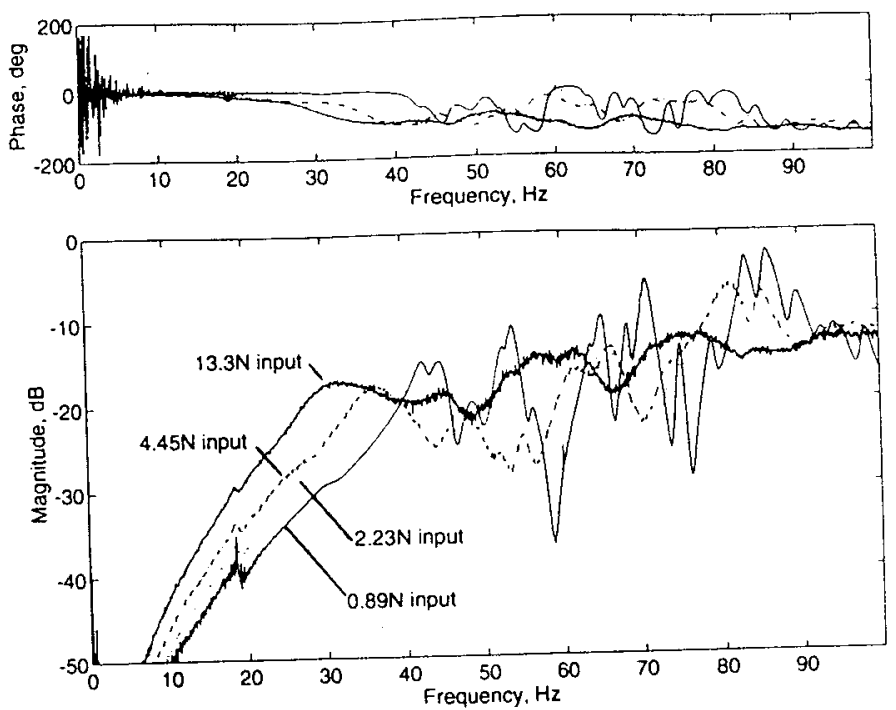

Figure 8: FRFs of Fully Deployed Structure for Multiple Input Amplitudes
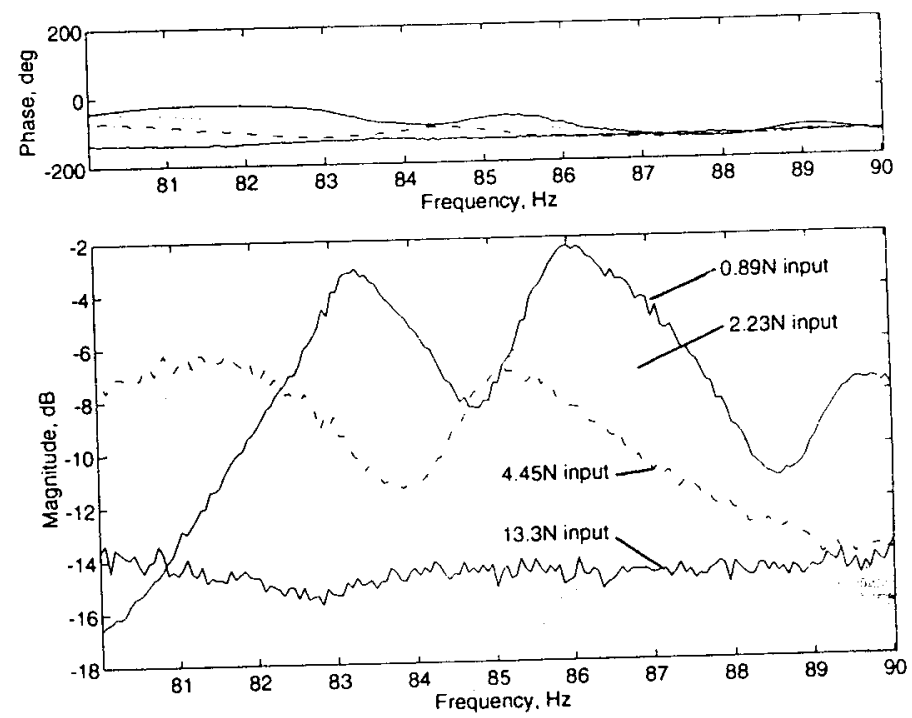

Figure 9: FRFs of Fully Deployed Structure for Multiple Input Amplitudes (Amplification of examined Modes A and B) 


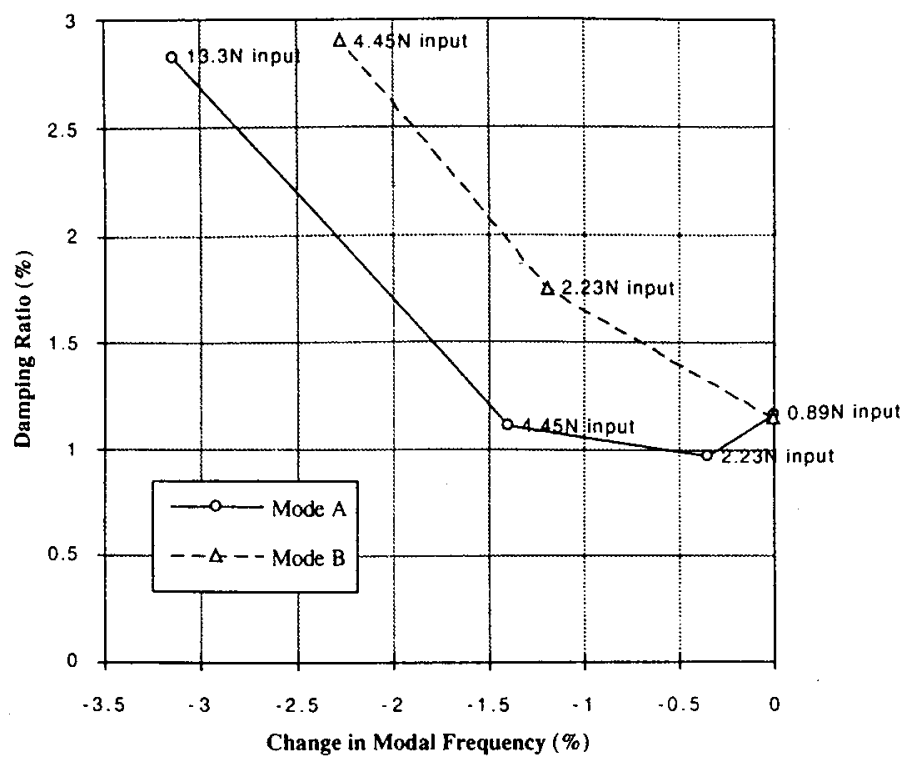

Figure 10: Modal Parameter Variations for Multiple Input Amplitudes

\section{Phase Plane Re Investigation Results}

Figure 11 displays a typical series of scans of the acceleration vs. input force phase plots. As can be seen from the equally scaled images, the shift into the obviously nonlinear state occurs over a relatively short increase in input amplitude. This bifurcation of resonance states was observed across the spectrum at varying levels of input amplitude.

Also noted during this part of the study was a hysteretic state path as a function of both input amplitude and frequency. This action was seen as the bifurcation would occur at one input amplitude as the amplitude was increased while the system would return to the previous state at a lower input amplitude as it was decreased. This hysteretic behavior also occurred upon increasing and decreasing the input frequency while the input amplitude was held constant.

During this experimentation, the question arose as to at what amplitudes these bifurcations were occurring at. The specifications for the pin-clevis joint parts suggested that free-play in the joints might occur near $15 \mu \mathrm{m}$. Recording the acceleration at which these bifurcations occurred at $5 \mathrm{~Hz}$ intervals across the spectrum allowed calculation of the maximum displacement of the structure at these points. Figure 12 portrays the results of this nonlinear boundary survey. These values proved to be repeatable to within $\pm 4 \mu \mathrm{m}$, but no correlation was evident between this boundary and the frequency response of the structure.
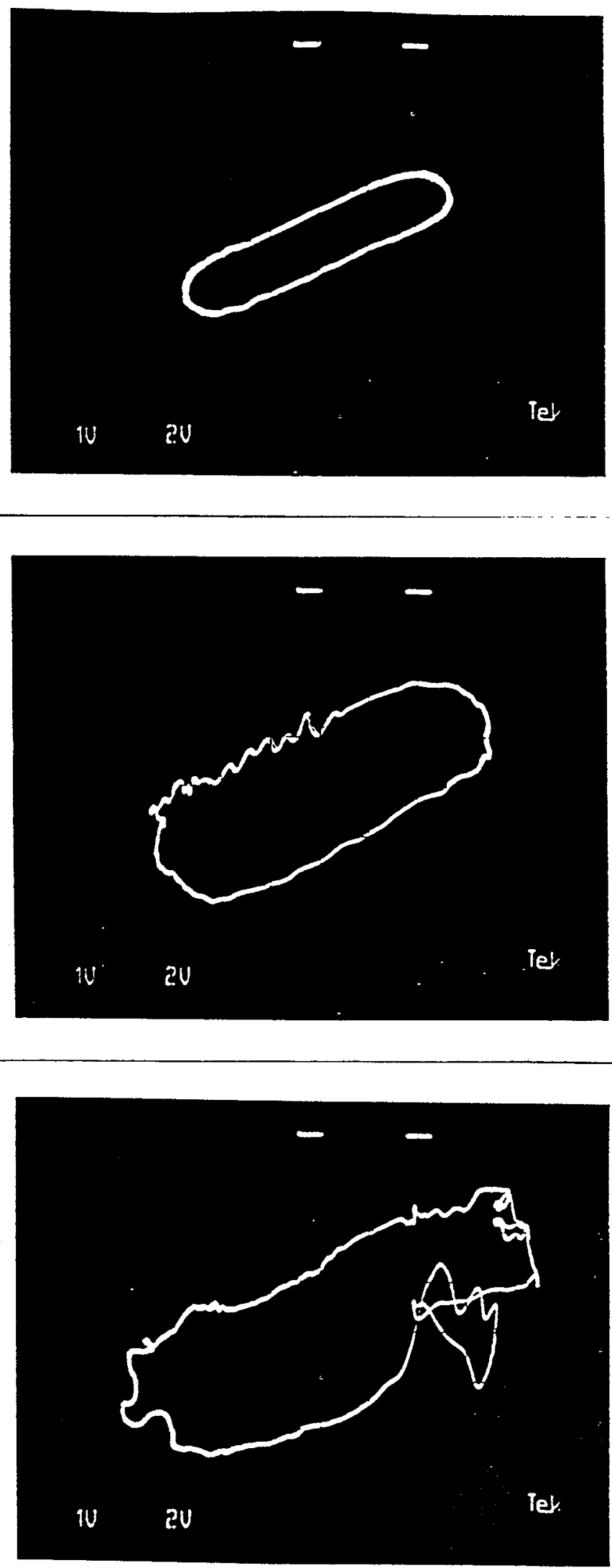

Figure 11: Phase Plane Bifurcation (Input force along the horizontal axes and acceleration along the vertical axes) 


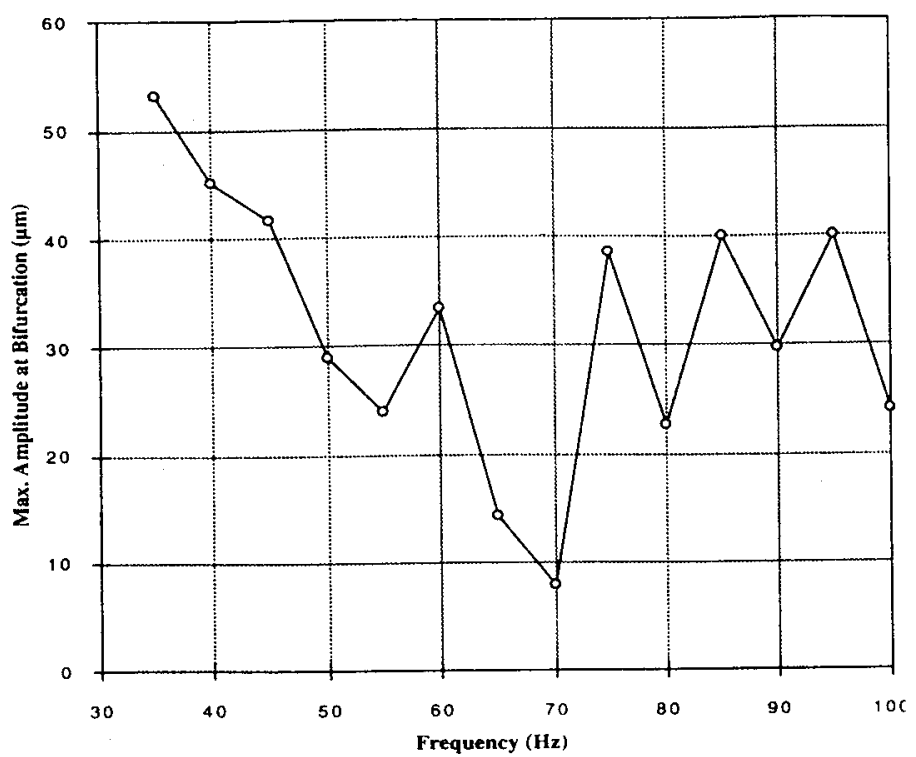

Figure 12: Map of the Nonlinear Boundary at Different Resonances

\section{CONCLUSIONS}

Modal displacements of the structure predicted by the finite element model portrayed unique mode shapes that are to be expected from such a compact deployable structure. The dependency of the dynamics of the structure on local displacements increased the influence of the nonlinear joint connections on the global response of the structure. Input amplitude dependent variations of modal frequencies and damping ratios indicated a dominance of nonlinear Coulomb friction. However, an examination of the phase plane response of the structure as input amplitude was increased revealed a hysteretic bifurcation of resonance states at a micron displacement level near the freeplay of the joints. The dominance of the Coulomb friction characteristics is most likely a result of the gravitational preloading of the joints increasing the friction while reducing the freeplay mechanics.

Further study of the structure under a different preloading condition is recommended for a better understanding of its influence. An attempt to model the structure with nonlinear considerations included is also recommended in order to determine the limits of our modeling accuracy.

\section{ACKNOWLEDGMENTS}

This work was supported by the Center for Space Construction (CSC) through NASA Grant No. NAGW-1388 and by NASA Langley Research Center (LaRC) through NASA Grant No. NAG-1-1490. Development of the test structure was supported by a grant from the Shimizu Corporation. The authors would like to acknowledge the assistance provided by Trudy Wittrock and Stephanie Gow, undergraduate research assistants, for their aid in the assembly of the finite element model and performance of the modal testing.

\section{REFERENCES}

1. Mikulas, M.M., and Withnell, P.R., "Construction Concepts for a Precision Segmented Reflector," Proceeding of the 34rd AIAA Structures, Structural Dynamics and Materials Conference, April, 1993, AIAA-93-1461.

2. Sarver, G.L. and Crawley, E.F., "Energy Transfer and Dissipation in Structures with Discrete Nonlinearities," Ph.D. Thesis, Dept. of Aeronautics and Astronautics, MIT, November, 1987.

3. Webster, M. and Velde, W.V., "Modelling Beam-Like Space Trusses with Nonlinear Joints with Application to Control," Ph.D. Thesis, Dept. of Aeronautics and Astronautics, MIT, June, 1991.

4. Bowden, M.L., "Dynamics of Structures with Nonlinear Joints," Ph.D. Thesis, Department of Aeronautics and Astronautics, MIT, May, 1988.

5. Crawley, E.F., Barlow, M.S., and van Schoor, M.C., "Variation in the Modal Parameters of Space Structures," Proceeding of the 33rd AIAA Structures, Structural Dynamics and Materials Conference, April, 1992, AIAA-92-2209.

6. Bullock, S.J., and Peterson, L.D., "Identification of Nonlinear Joint Mechanics for a Deployable Joint," Proceedings of the 35th AIAA Structures, Structural Dynamics and Materials Conference, April, 1994, AIAA-94-1347.

7. Juang, J.-N. and Pappa, R.S., "An Eigensystem Realization Algorithm for Modal Parameter Identification and Model Reduction," Joumal of Guidance, Control and Dynamics, Vol. 8, Sept.-Oct. 1985, pp. 620-627.

8. Alvin, K., Park, K.C. and Belvin, W., "A Second-Order Structural Identification Procedure via State Space-Based System Identification," Proceedings of the 1992 AIAA Guidance, Navigation, and Control Conference, Hilton Head Island, August 1992, AIAA-92-4387.

9. MSC/NASTRAN User's Manual, The MacNeal-Schwendler Corporation, Version 67, August, 1991, ISSN 0741-8019.

10. LabVIEW 2 User Manual, National Instruments Corporation, September 1991 Edition, Austin, TX. 